The perception of behaviour associated with dementia in the acute hospital

Zumer Jawaid ${ }^{1 \star}$, George Crowther ${ }^{2}$ and Syeda Ashar ${ }^{1}$

${ }^{1}$ Leeds and York Partnership Foundation Trust and ${ }^{2}$ Leeds Teaching Hospitals Trust

${ }^{*}$ Corresponding author.

doi: 10.1192/bjo.2021.693

Aims. General hospital based Health Care Professionals (HCPs) use very varied language to describe behaviour in dementia. Lessons from medicine and other professions tell us that nonuniform communication is a source of error and subsequent poor decision making. Knowing how HCPs communicate behaviour in dementia in a hospital setting may help better understand these potential sources of communication error and identify training needs.

Background. Around 25\% of hospital beds occupied with people living with dementia. Hospitalised patients with dementia have a high prevalence of distressing symptoms (pain 70\%, delirium $66 \%$, depression $35 \%$, anxiety $34 \%$, hallucinations $14 \%$ delusions $11 \%)$. These symptoms often displayed as behaviour can be challenging for HCPs to interpret. Variations in communicating behaviour may lead to inconsistent understanding of the need, with the potential for missing treatable conditions that drive the behaviour. Standardizing communication and documentation have the potential to improve the quality of information handed over between HCPs which may improve the quality of care and patient outcomes.

Method. Qualitative methodology including photo elicitation was used. A purposive sample of 59 HCPs was selected. This was identified from a range of professional backgrounds, experience levels and medical specialities. They were presented with a photograph and case vignettes depicting 4 behaviours associated with distress (aggression, depression, delirium and psychosis). HCPs were asked to respond to the scenarios as if they were handing over to colleagues or documenting in the medical record. Data were analysed by thematic analysis.

Result. 59 HCPs were interviewed with photo-elicitation. Participants recorded their responses in limited time to reflect time constraints in a busy ward environment. 2 HCPs declined to participate in research.

When describing behaviour associated with aggression and depression HCPs were consistent with the language used (49/57). When presented with a delirium less consistency was observed (31/47). While describing psychosis each HCP chose either paranoia or suspiciousness among other descriptions.

Conclusion. Overall there has been consistency in describing the distress experienced by the patient even though HCPs came from very different roles and specialities. Doctors, Nurses, CSWs and dieticians all described the behaviour alike. Newer staff were more accurate which could be due to dementia training within National Dementia Action Alliance.

\section{Course and outcome of comorbid mood disorders in children and adolescents with intellectual disability [IDD]}

Arul Jayendra Pradeep Velusamy ${ }^{1 *}$, Satish Chandra Girimaji ${ }^{2}$ and John Vijay Sagar Kommu ${ }^{2}$

${ }^{1}$ Former Senior resident, National Institute of Mental Health and Neuro Sciences [NIMHANS], RCPsych MTI Trainee, Central and North West London NHS Trust and ${ }^{2}$ Professor, Department of Child and Adolescent Psychiatry, National Institute of Mental health and Neuro Sciences [NIMHANS]

${ }^{*}$ Corresponding author.

doi: 10.1192/bjo.2021.694

Aims. The primary objective of the study is to assess the clinical course and functional outcome of comorbid mood disorders in children and adolescents with Intellectual Disability [IDD]

Method. 53 children and adolescents with varying levels of severity of IDD presenting with comorbid mood disorders diagnosed using Kiddie Schedule for Affective Disorder and SchizophreniaDSM-5 version [KSADS] were recruited by convenient sampling with the exclusion of autism spectrum disorders. Vineland Social Maturity Scale [VSMS] is used to quantify the severity of ID. Developmental Behaviour Checklist-Parent [DBC-P] version is used to measure psychopathology, Clinical Global severity of illness [CGI-S] to quantify the clinical improvement, and Developmental Disabilities Children's Global Assessment of Severity [DD-CGAS] to assess functional improvement. Prospective naturalistic follow-up was done with assessment points at baseline, 1, 3- and 6-month timeline. Result. 40 patients were followed up for 6 months period. Overall significant improvement is observed in the dependent variables like CGI, DDCGAS, and DBC-P from baseline to 3 months and then a plateau of improvement from 3 to 6 months. The diagnostic breakup of mood disorders is mania $[\mathrm{N}=19]$, Depression $[\mathrm{N}=12]$, and mixed affective state $[\mathrm{N}=9]$. Patients with mania had significant improvement in DBC score $[\mathrm{F}=12.69, \mathrm{p}<0.001$ in repeated measures ANOVA], DDCGAS [p $<0.001$ ], and CGI score $[p<0.03$ ] with an overall remission rate of $42.10 \%$ over 6 months period. Patients with depression had significant improvement in DBC score $[\mathrm{F}=15.48, \mathrm{p}<0.001]$, DDCGAS, and CGI score $[\mathrm{p}<0.001]$ with an overall remission rate of $41.7 \%$. None of the patients with mixed affective states had clinical remission with no significant improvement observed in any of the dependent variables measuring course and outcome.

Conclusion. Comorbid psychiatric disorders in children and adolescents with IDD have a guarded prognosis compared to mood disorders in neurotypical children. Comorbid ADHD and caregiver stress majorly influenced the course and outcome in the current study.

Post-traumatic stress disorder (PTSD), anger and mental health of school students in Syria after nine years of conflict: a large-scale school-based study

Sami Jomaa ${ }^{1 *}$, Ameer Kakaje ${ }^{1}$, Ragheed Al Zohbi ${ }^{2}$, Ayham Alyousbashi' ${ }^{1}$, Rawan N K Abdelwahed ${ }^{1}$, Osama Hosam Aldeen ${ }^{1}$, Mohammad Marwan Alhalabi ${ }^{1}$, Ayham Ghareeb ${ }^{1}$ and Youssef Latifeh ${ }^{3}$

${ }^{1}$ Faculty of Medicine, Damascus University; ${ }^{2}$ Faculty of Medicine, Aleppo University and ${ }^{3}$ Psychiatry Department, Al-Mowasat University Hospital, Department of Internal Medicine, Syrian Private University

${ }^{*}$ Corresponding author.

doi: 10.1192/bjo.2021.695

Aims. The Syrian crisis has entered its ninth year with many being affected by the war. This is the largest-scale study that aims to evaluate the psychological profile of secondary school students in Syria. Method. This is a cross-sectional study in schools in Damascus, Syria. The surveys assessed working habits, smoking, war exposure, grades, socioeconomic status (SES), social support, health-related quality of life (HRQL), post-traumatic stress disorder (PTSD), problematic anger, and other parameters. 